\title{
HYPERTREHALOSAEMIC AND HYPERLIPAEMIC RESPONSES TO ADIPOKINETIC HORMONE IN FIFTH LARVAL INSTAR LOCUSTS, LOCUSTA MIGRATORIA
}

\author{
W. J. A. van MarrewiJk, A. Th. M. van den Broek, D. J. van der Horst \\ and A. M. TH. BeENAKKERS \\ Laboratory of Chemical Animal Physiology, State University of Utrecht, 8 Padualaan, 3508 TB Utrecht, \\ The Netherlands
}

(Received 23 May 1983)

\begin{abstract}
In fifth instar larvae of Locusta migratoria the haemolymph lipid concentration is elevated after injection of adipokinetic hormone (AKH). This hyperlipaemic response in larvae remains substantially lower than in adults; over $75 \%$ of the mobilized lipid consists of diacylglycerol. In addition, unlike adult locusts, fifth instar larvae also exhibit a consistent, though moderate, hypertrehalosaemic response to $\mathrm{AKH}$. The increases of both lipid and carbohydrate concentrations in larvae are dose-dependent, showing a significant linear regression on log dose in the range $0.2-20 \mathrm{pmol} \mathrm{AKH}$.

Glycogen phosphorylase in the fat body of fifth instar larvae as well as young adults is activated on injection of $\mathrm{AKH}$, the percentage active phosphorylase increasing linearly with log dose in the range 0.04-20 pmol AKH. For a given response, a somewhat higher dose of AKH is needed in larvae than in young adults.

Fat body glycogen phosphorylase is strongly activated during the period of the larval-adult ecdysis, when active phosphorylase accounts for almost half of the total enzyme, which is approximately ten times more than it is two days before, and two days after the ecdysis.

The corpora cardiaca of fifth larval instar locusts already possess the potencies to elevate carbohydrate and lipid concentrations in larval haemolymph, and to activate fat body glycogen phosphorylase.
\end{abstract}

Key Word Index: Locusta migratoria, larvae, hypertrehalosaemia, hyperlipaemia, glycogen phosphorylase, adipokinetic hormone, corpus cardiacum, fat body

\section{INTRODUCTION}

Locust flight is known to raise diacylglycerol and to reduce trehalose concentrations in haemolymph, both compounds reaching steady state levels during sustained flight. These substrates are derived from triacylglycerol and glycogen stores in the fat body respectively and provide energy for the flight muscles. Extracts from corpora cardiaca (CC), and synthetic adipokinetic hormone (AKH) evoke hyperlipaemia in locusts also, but they have no influence on haemolymph carbohydrate levels (for reviews, see Beenakkers et al., 1981a, b, 1983). However, in the locust fat body flight activity results in the activation of glycogen phosphorylase, the enzyme determining the rate of glycogenolysis (Van Marrewijk et al., 1980a). This phosphorylase activation is needed to meet the enhanced turnover rate of haemolymph trehalose during sustained flight (Van der Horst et al., 1978). Fat body phosphorylase is also activated upon injection of CC-extracts (Beenakkers et al., 1978), and recently this effect of the $\mathrm{CC}$ on phosphorylase was shown to be due virtually entirely to AKH (Van Marrewijk and Beenakkers, 1982; Van Marrewijk et al., 1983).

In last larval instar locusts, at a stage when flight capacity is still developing (Beenakkers et al., 1975; Van Marrewijk et al., 1980b), the CC already contain AKH (Goldsworthy et al., 1973). Moreover, in the larval fat body, as in adults, the concentration of cyclic AMP is enhanced upon injection of CCextracts or AKH (Gäde and Beenakkers, 1977). However, larval locusts exhibit only an incomplete adipokinetic response to CC-extracts compared to adults (Mwangi and Goldsworthy, 1977). Because of this limited effect on lipid release, involvement of AKH in the regulation of carbohydrate metabolism in developing locusts might be anticipated.

In the present report the hormonal effects on carbohydrate and lipid concentrations in larval haemolymph are quantified. In addition, the possible role of $\mathrm{AKH}$ in the control of glycogenolysis in the larval fat body is investigated.

\section{MATERIALS AND METHODS}

Locusts (Locusta migratoria) were reared in the laboratory under crowded conditions at $30^{\circ} \mathrm{C}, 40 \%$ r.h. and a photoperiod of $12 \mathrm{hr}$. The insects were fed on a diet of floating-grass supplemented with rolled oats. Under the conditions described, the fifth larval instar period lasted about ten days. Males from the fifth larval instar and from the adult stage were used for experimentation.

Preparation and injection of gland extracts

Corpora cardiaca (CC) of five-day-old fifth instar larvae or of twelve-day-old mature adults were dissected free, rinsed, and placed in insect saline $(128 \mathrm{mM} \mathrm{NaCl}, 5 \mathrm{mM}$ $\mathrm{KCl}$ ). Cells were ruptured by six freeze-thaw cycles. The 
resulting suspension was boiled for $3 \mathrm{~min}$, cooled, and centrifuged at $12,000 \mathrm{~g}$ for $5 \mathrm{~min}$ at $3^{\circ} \mathrm{C}$. The supernatant was used as CC-extract and, at a concentration of $0.1 \mathrm{CC}$ pair eq $/ 10 \mu \mathrm{l}$, stored at $-20^{\circ} \mathrm{C}$. Each extract was prepared from the pooled $\mathrm{CC}$ of at least six individuals.

CC-extracts and solutions of synthetic AKH (Peninsula Laboratories, San Carlos, California, U.S.A.) in saline were injected in a $10 \mu \mathrm{l}$ volume into the abdominal cavity, ventrally between the first and second abdominal segments. After injection, locusts were kept individually at $30^{\circ} \mathrm{C}$.

\section{Determination of phosphorylase activity}

For isolation of fat body the abdomen was cut off after the first segment, the head with the attached gut was removed, and fat body tissue was quickly dissected free from the thorax and the first abdominal segment. The tissue was homogenized by hand with a chilled glass homogenizer (Teflon pestle) in $1 \mathrm{ml}$ of an ice-cold buffer medium ( $\mathrm{pH} 7.0$ ) containing $50 \mathrm{mM}$ phosphate, $5 \mathrm{mM}$ EDTA, and $20 \mathrm{mM}$ $\mathrm{NaF}$. The tissue homogenate was centrifuged at $35,000 \mathrm{~g}$ for $15 \mathrm{~min}$ at $0^{\circ} \mathrm{C}$ and the layer between the precipitate and the floating fat was immediately used for the enzyme assay. Glycogen phosphorylase activity was measured spectrophotometrically in the direction of glycogen degradation as described previously (Van Marrewijk et al., 1980a). Active phosphorylase was assayed in the absence of 5'AMP, whereas total phosphorylase was measured in the presence of $2 \mathrm{mM} S^{\prime}$-AMP. Hormonal effects on phosphorylase activity were measured $15 \mathrm{~min}$ after injection of CC-extracts or synthetic AKH. Protein in the fat body extracts was determined by the Lowry method as modified by Schackterle and Pollack (1973) using bovine serum albumin (Fluka AG, Buchs, Switzerland) as a standard.

\section{Determination of carbohydrate and lipid in haemolymph}

Haemolymph (about $10 \mu \mathrm{l} /$ locust) was sampled with a microsyringe from a puncture ventrally in the neck membrane. Samples were centrifuged at $12,000 \mathrm{~g}$ for $0.5 \mathrm{~min}$ at $3^{\circ} \mathrm{C}$, and aliquots $(5-10 \mu 1)$ from the supernatants were added to $1.5 \mathrm{ml} 18 \mathrm{M}$ sulphuric acid. Total carbohydrate and lipid contents in the stirred mixtures were determined.

Carbohydrate was estimated by the modified anthrone method of Spik and Montreuil (1964), with trehalose (BDH, Poole, England) as a standard.
Total lipids were quantified by the vanillin method of Zöllner and Kirsch (1962). For the determination of the haemolymph lipid composition the extraction of total lipid and the separation and quantification of individual lipid classes were performed as described by Van der Horst et al. (1983).

\section{Statistical treatment of results}

Regression of increases in haemolymph carbohydrate and lipid levels and of phosphorylase activation on the hormonal dose was computed by analysis of variance and regression analysis according to Sokal and Rohlf (1969). Regression lines were compared by analysis of covariance as described by Snedecor and Cochran (1967). For comparisons between treatments the Wilcoxon two-sample test was used (Sokal and Rohlf, 1969). Probability less than 95\% was considered non-significant.

\section{RESULTS}

\section{AKH-induced hypertrehalosaemia and hyperlipaemia}

Figure 1 shows the time course of changes in haemolymph carbohydrate and lipid concentrations in five-day-old fifth instar larvae upon injection of AKH. The hormone induced an increase in concentration of both substrates, the response being maximal around $90 \mathrm{~min}$ after $\mathrm{AKH}$-injection. After $3 \mathrm{hr}$, the lipid concentration had returned to its control level, whereas the carbohydrate concentration was still elevated. In further experiments for measuring hormone-induced changes in haemolymph substrate levels an incubation time of $90 \mathrm{~min}$ was chosen, enabling us to estimate near-maximal responses to AKH of both carbohydrate and lipid in haemolymph of the same animals.

Figure 2 shows that AKH induces a dosedependent increase of haemolymph lipid and carbohydrate levels in mid fifth instar larvae (A, B) as well as shortly before the final moult $(C, D)$. A significant linear regression $(P<0.001$ in all cases) of the increases in lipid and carbohydrate levels on log dose

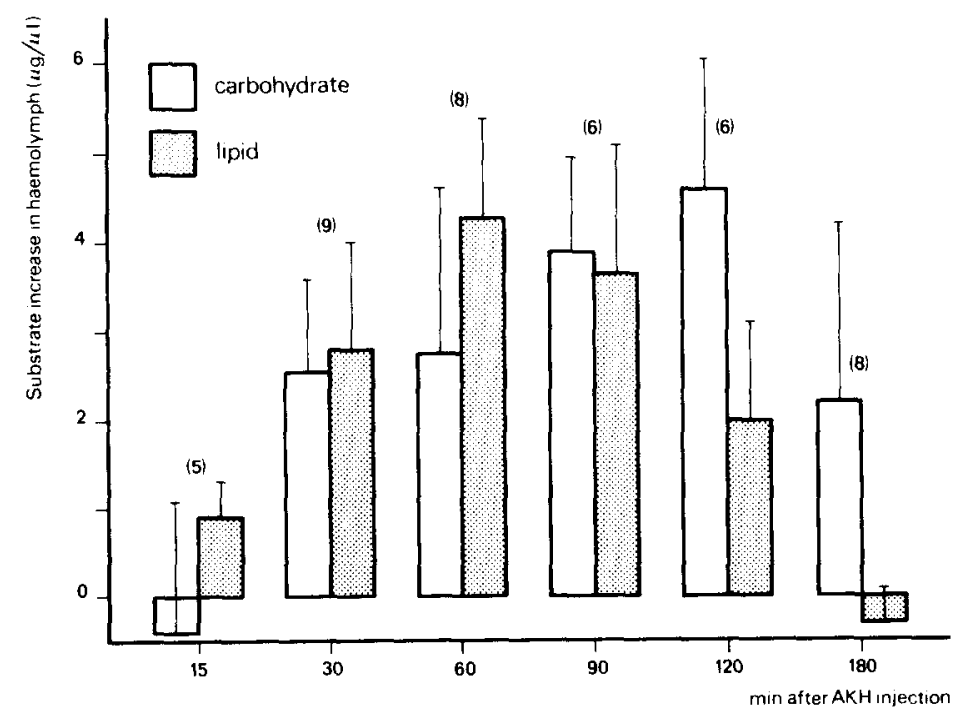

Fig. 1. Time course of the changes of carbohydrate and lipid concentrations in haemolymph of five-day-old fifth instar larvae of Locusta migratoria after the injection of 10 pmol synthetic AKH. Data presented are changes after the subtraction of saline control values. Means and SD are shown for the number of individuals in parentheses. 


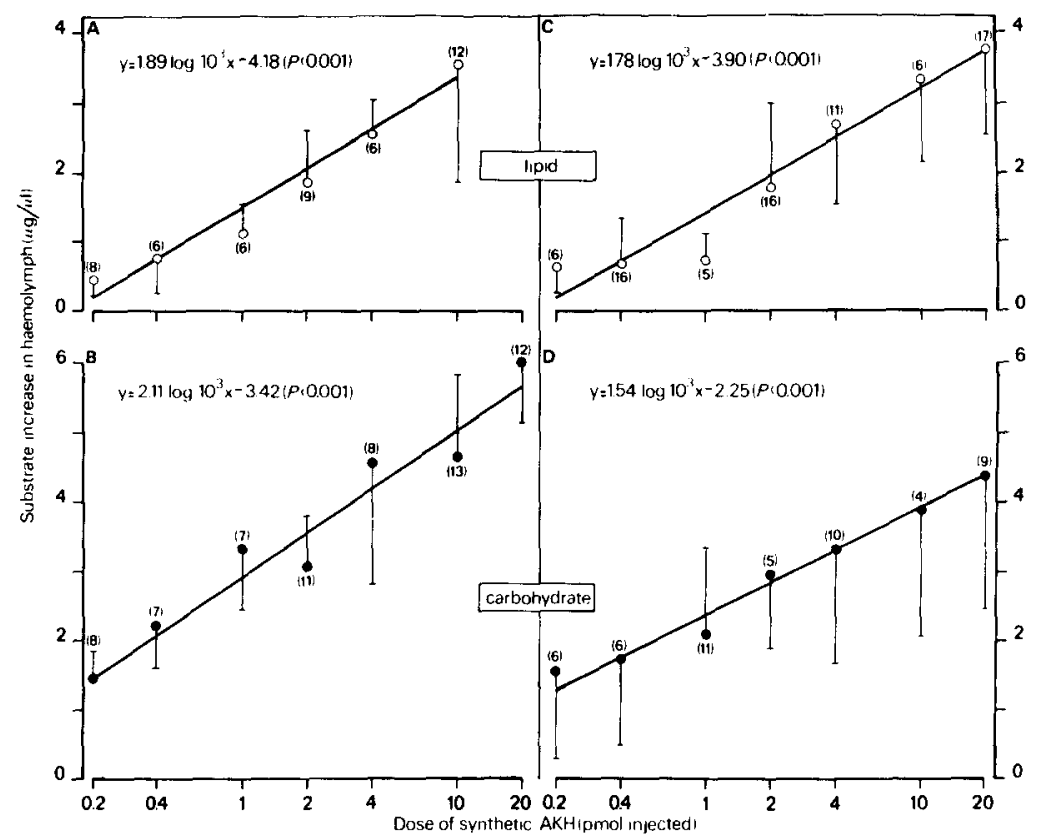

Fig. 2. Dose-response relationships for the changes of carbohydrate and lipid levels in haemolymph of five-day-old (A, B) and eight-day-old (C, D) fifth instar larvae of Locusta migratoria upon injection of synthetic AKH. Values presented are the results after subtraction of the control values obtained upon saline injection. Lines represent a best fit of the data as obtained by regression analysis. Regression equations and significance of regression are also shown. Values are means $\pm S D$ for the number of individuals in parentheses.

was observed in the range $0.2-10 \mathrm{pmol}(\mathrm{A})$ or 0.2 to $20 \mathrm{pmol}$ AKH (B, C, D). In untreated five and eight-day-old fifth instar larvae the mean haemolymph concentrations of lipid were 4.6 and $6.0 \mu \mathrm{g} / \mu \mathrm{l}$, respectively, and of carbohydrate 17.4 and $22.4 \mu \mathrm{g} / \mu \mathrm{l}$, respectively. Thus, the maximal increases shown in Fig. 2 correspond to $70 \%$ (C) and $90 \%$ (A) of the control values for lipid, and to $20 \%$ (D) and $30 \%$ (B) for carbohydrate.

The changes in the composition of the haemolymph lipid fraction of fifth instar larvae upon injection of AKH are shown in Table 1. Diacylglycerols appear to be responsible for by far the major part of the hormone-induced hyperlipaemia in larvae, with only minor contributions from other lipid components.
To establish the presence of hypertrehalosaemic along with hyperlipaemic activity in larval CC, CCextracts from fifth instar larvae were injected in insects of the same age (Table 2). The CC-extracts caused an increase of both carbohydrate and lipid concentrations in haemolymph. The magnitude of the responses of both substrates to the larval CC-extract did not differ significantly from the responses to a corresponding amount ( 0.01 pair eq) of CC-extracts from mature adults, also shown in Table 2 .

\section{Hormonal activation of fat body glycogen phos-} phorylase

The activities of total phosphorylase in fat body of fifth instar larvae and adult locusts are shown in Table 3. The specific activity of the enzyme was

Table 1. The effect of AKH on the composition of haemolymph lipids in eight-day-old fifth instar larvae of Locusta migratoria. The lipid composition was estimated prior to, and $90 \mathrm{~min}$ after the injection of $10 \mathrm{pmol}$ synthetic AKH

\begin{tabular}{lcccc}
\hline & \multicolumn{2}{c}{ Before injection } & \multicolumn{2}{c}{ After injection } \\
\cline { 2 - 4 } Lipid fraction & $\mu \mathrm{g} / \mu \mathrm{l}$ & $\%$ of total & $\mu \mathrm{g} / \mu \mathrm{l}$ & $\%$ of total \\
\hline Phospholipid & $0.8 \pm 0.2$ & 13 & $1.0 \pm 0.1$ & 9 \\
Triacylglycerol & $0.9 \pm 0.3$ & 14 & $0.8 \pm 0.3$ & 8 \\
Diacylglycerol & $1.9 \pm 0.7$ & 31 & $5.2 \pm 1.4$ & 49 \\
Free fatty acid & $1.0 \pm 0.2$ & 16 & $1.4 \pm 0.2$ & 13 \\
Sterol & $0.6 \pm 0.2$ & 10 & $0.9 \pm 0.2$ & 9 \\
Monoacylglycerol & $0.3 \pm 0.1$ & 5 & $0.4 \pm 0.1$ & 4 \\
Sterol ester & $0.7 \pm 0.2$ & 11 & $0.8 \pm 0.2$ & 8 \\
Total & $6.2 \pm 0.9$ & & $10.5 \pm 1.5$ & \\
\hline
\end{tabular}

Means for pooled haemolymph of three groups of 15 animals each are shown as absolute amounts ( $\mu \mathrm{g}$ lipid $/ \mu 1$ haemolymph) $\pm \mathrm{SD}$, and as percentages of total lipid content. 
Table 2. Hypertrehalosaemia and hyperlipaemic effects of corpus cardiacum (CC) extracts from five-day-old fifth instar larvae (5d-L5) and from twelve-day-old mature adults (12d-Ad) of Locusta migratoria upon injection into 5d-L5 acceptor locusts

\begin{tabular}{lcccc}
\hline & \multicolumn{2}{l}{$\begin{array}{l}\text { Increase of haemolymph concentration } \\
\text { Carbohydrate }\end{array}$} & \multicolumn{2}{c}{ Lipid } \\
CC-extract from & $\mu \mathrm{g} / \mu \mathrm{l}$ & $\%^{*}$ & $\mu \mathrm{g} / \mu \mathrm{l}$ & $\%$ \\
5d-L5, 0.01 pair eq & $3.5 \pm 1.8$ & 21 & $3.6 \pm 1.4$ & 70 \\
$12 \mathrm{~d}-\mathrm{Ad}, 0.01$ pair eq & $4.4 \pm 1.4$ & 26 & $4.0 \pm 1.3$ & 79 \\
\hline
\end{tabular}

*Percentages of haemolymph concentrations before injection. Means + SD for seven individuals are shown. Differences between Sd-L5 and 12d-Ad are not significant.

higher in adults than in larvae $(P<0.01$ for two-dayold adults compared to both five and eight-day-old larvae). This increase of total phosphorylase activity must have preceded ecdysis, as at that time the activity does not differ from that of two-day-old adults, but is much higher than that of eight-day-old larvae $(P<0.01)$. Throughout the adult stage differences between the activities in young and mature adults were not significant. At the incubation time used for measuring hormonal activation of phosphorylase (15 min after hormone injection) total phosphorylase activity was not affected by AKH.

Glycogen phosphorylase in the fat body of last instar larvae as well as young adult locusts could be activated by AKH (Fig. 3). In both developmental stages phosphorylase activation was dose-dependent; the increase of active phosphorylase exhibited a significant linear regression on log dose in the range 0.04-20 pmol synthetic $\mathrm{AKH}$, according to the regression equations shown in Fig. 3. Comparison of the regression lines by analysis of covariance showed that the slopes (regression coefficients) did not differ, whereas the elevations of the two lines were significantly different $(P<0.02)$. This indicates that in larvae the amount of $\mathrm{AKH}$ needed for a given response is somewhat higher than in adult locusts.

Table 4 shows the effect of the injection of extracts from larval $\mathrm{CC}$ on phosphorylase activity in the

Table 3. Activity of total phosphorylase $(a+b)$ in locust fat body during fifth larval instar (L5) and adult (Ad) development

\begin{tabular}{cc}
\hline Age & Activity* \\
5-Day-old L5 & $3.4 \pm 0.6(11)$ \\
8-Day-old L5 & $2.3 \pm 0.6(21)$ \\
L5 $\rightarrow$ Ad ecdysis & $6.7 \pm 1.9(6)$ \\
2-Day-old Ad & $6.8 \pm 1.9(15)$ \\
4-Day-old Ad & $7.7 \pm 1.7(18)$ \\
12-Day-old Ad & $8.4 \pm 2.3(39)$ \\
\hline *Activities are expressed as $\mu$ mol glycosyl \\
hr $^{-1}$ mg protein ${ }^{-1}$, and are means \pm SD for \\
the number of individuals in parentheses.
\end{tabular}

larval fat body. Over $50 \%$ of total phosphorylase was found in the active form. The response to 0.005 pair eq CC-extract already approached the maximum value normally found for hormone-induced phosphorylase activation ( $60-65 \%$ active phosphorylase). As a consequence, the activation induced by 0.1 pair eq CC-extract was not significantly higher. Thus, the CC of fifth larval instar locusts already possess a high capacity to activate fat body glycogen phosphorylase.

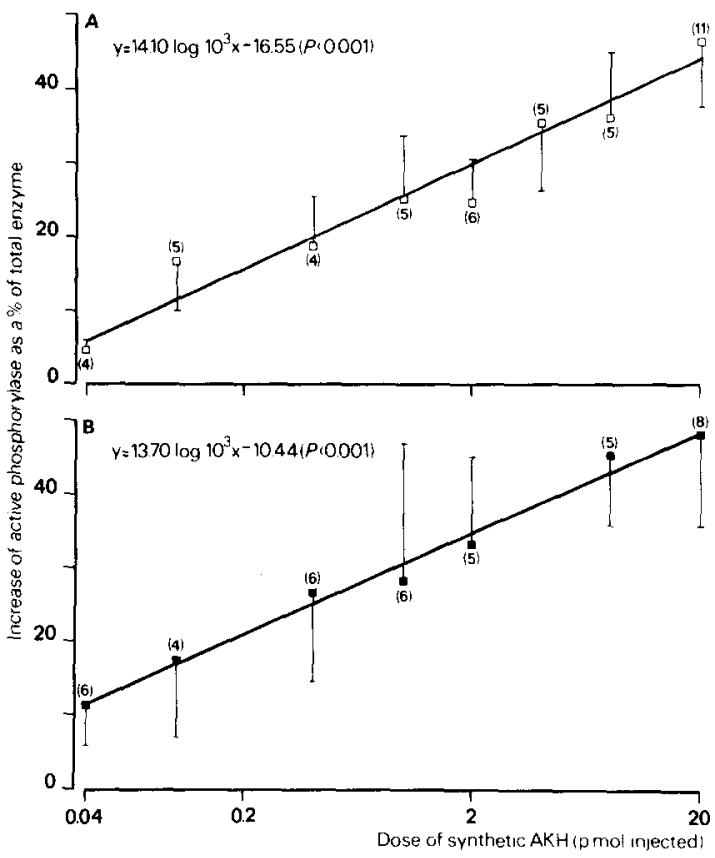

Fig. 3. Dose-response relationships for the activation by synthetic AKH of glycogen phosphorylase in the fat body of eight-day-old fifth instar larvae (A) and two-day-old adults (B) of Locusta migratoria. For details see legend to

Fig. 2.

Table 4. Activation of glycogen phosphorylase in the fat body of five-day-old larval locusts upon injection of CC-extracts from larvae of the same age

\begin{tabular}{lcc}
\hline & $\begin{array}{c}\text { Activation } \\
\text { state } \\
(\%)^{*}\end{array}$ & $\begin{array}{c}\text { Difference } \\
\text { from saline }\end{array}$ \\
Treatment & $6.7 \pm 1.2(6)$ & $P<0.05$ \\
No injection & $13.0 \pm 5.4(5)$ & \\
Saline & $57.8 \pm 9.6(5)$ & $P<0.02$ \\
CC-extract, 0.005 pair eq & $62.0 \pm 7.7(6)$ & $P<0.02$ \\
CC-extract, 0.1 pair eq &
\end{tabular}

*Values represent active phosphorylase (\% of total enzyme) and are the means \pm SD for the number of individuals in parentheses. 


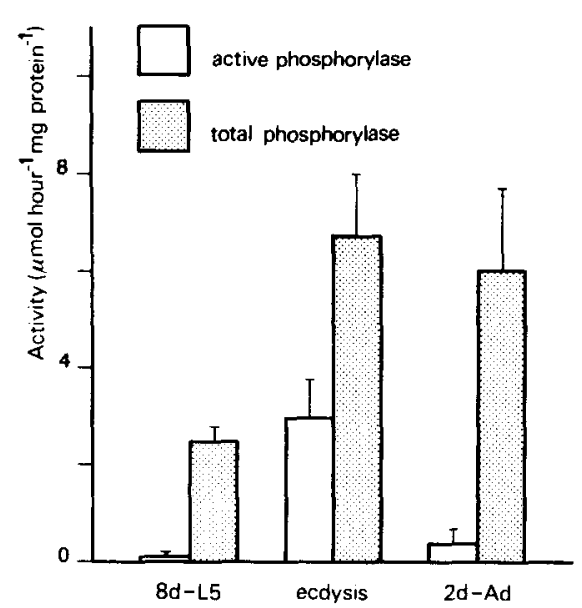

Fig. 4. Active and total phosphorylase activity in the locust fat body during the larval-adult ecdysis compared to the activities in late larvae two days before $(8 \mathrm{~d}-\mathbf{L} 5)$, and in young adults two days after the ecdysis (2d-Ad). Values are means and SD for 6 or 7 individuals.

The activity of phosphorylase in the locust fat body during the larval-adult moult, at the time of ecdysis (the period of the shedding of the old cuticle), is shown in Fig. 4. The enzyme was highly activated during the ecdysis, with $45 \%$ of total phosphorylase in the active form, compared to only $4 \%$ in late larvae two days before, and $6 \%$ in young adults two days after the ecdysis.

\section{DISCUSSION}

Locust adipokinetic hormone causes an elevation of haemolymph lipid levels in adult locusts. In addition to this hyperlipaemic effect AKH possesses hypertrehalosaemic potency, as has been shown upon AKH injection into cockroaches (Goldsworthy et al., 1972; Jones et al., 1977). Adult locusts, however, do not respond to $\mathrm{AKH}$ with hypertrehalosaemia.

The results of the present study show that in fifth instar larval locusts AKH not only raises haemolymph lipid levels, but elevates carbohydrate concentrations as well. As haemolymph carbohydrate in larval locusts consists almost entirely of trehalose (Hill and Goldsworthy, 1968), it seems obvious that the increased carbohydrate levels also represent a hypertrehalosaemic response. For both carbohydrate and lipid the magnitude of the response to $\mathrm{AKH}$ appears to depend on the hormonal dose over a rather wide range (Fig. 2), only the lower part of which might represent physiological concentrations. A similar dose-dependence has been observed for the hypertrehalosaemic activity of shrimp red pigmentconcentrating hormone, locust AKH and "Compound II" in Periplaneta, carbohydrate increases in haemolymph exhibiting linear regression on log hormonal dose over a wide range (Van Norstrand et al., 1980). With AKH, this linear relationship was maintained from 0.015 to no less than 150 pmol, so over a range covering four orders of magnitude.

If corpus cardiacum extracts from mid fifth instar larvae are injected into larvae of the same age, lipid and carbohydrate concentrations in the haemolymph are elevated (Table 2). Obviously, the $\mathrm{CC}$ of fifth instar larval locusts already possess both hyperlipaemic and hypertrehalosaemic potencies, the former being comparable to that of adult CC. CCextracts from fifth instar larvae induce hyperlipaemia in adult locusts as well (Goldsworthy et al., 1973; Gäde and Beenakkers, 1977), indicating the presence of $\mathrm{AKH}$ in larval $\mathrm{CC}$.

A moderate adipokinetic response of fifth instar larvae to $C C$-extracts ( 0.01 pair eq) from adult locusts has also been reported by Mwangi and Goldsworthy (1977). In contrast, a hypertrehalosaemic effect as shown in the present study was entirely absent in the experiments of Gäde and Beenakkers (1977), who injected larvae with a high dose ( 0.1 pair eq) of larval $\mathrm{CC}$. The reason for this discrepancy may reside in the use by the latter authors of female locusts with haemolymph carbohydrate levels almost twice as high as in the male locusts used in the present study (about 35 and $20 \mathrm{mg} / \mathrm{ml}$, respectively).

The adipokinetic response in larvae is mainly caused by an elevation of the diacylglycerol concentration (Table 1). This fraction, constituting half of the total lipid content in the haemolymph of AKHinjected larvae, is responsible for over $75 \%$ of the hormone-induced lipid increase. Approximately 10\% of the lipid increase in larval haemolymph might be due to free fatty acids. Thus, the hormone-induced changes in the haemolymph lipid pattern of larvae appear to be very similar to the changes observed in adult locusts (Mayer and Candy, 1969; Beenakkers, 1973). However, the magnitude of the adipokinetic response in larvae remains substantially below the level reached in adults. The reason for this incomplete response is neither a shortage of lipid in the larval fat body (Hill and Goldsworthy, 1968) nor a lack of response of the fat body to the hormone, since AKH elicits in larvae, like in adults, an elevation of the cyclic AMP concentration in the fat body (Gäde and Beenakkers, 1977). A more likely explanation for the poor adipokinetic response, as has been suggested by Mwangi and Goldsworthy (1977), could be the inability in larvae to form lipoprotein $\mathrm{A}^{+}$in the haemolymph, which in adult locusts is essential for the binding of the increased amount of the diacylglycerols released after AKH-injection. Indeed, Wheeler and Goldsworthy (1983) observed no increase in heparin-EDTA precipitable proteins, which they used to estimate lipoprotein $\mathrm{A}^{+}$, after injection of glandular lobe extracts throughout the fifth larval instar. In our laboratory we have found by gel filtration chromatography an $\mathrm{A}^{+}$like protein fraction which carries the increased diacylglycerol in fifth instar larvae; the possible identity with adult $\mathbf{A}^{+}$and the relation between the adipokinetic response and the haemolymph (lipo)protein pattern in developing locusts is currently under investigation.

In addition to the increase of carbohydrate and lipid levels in the haemolymph of fifth instar larvae upon AKH-injection, there is also a dose-dependent activation of glycogen phosphorylase in the larval fat body (Fig. 3). This activation is due entirely to the conversion of the inactive form of the enzyme to the active form, the activity of total phosphorylase being 
unaffected by the hormone, as expected in view of the short incubation time (15 min). The lower specific activity of total phosphorylase in larvae compared to adults (Table 3 ) not necessarily reflects a corresponding lower enzyme content of the larval fat body. The difference can, at least partly, be explained by the variations in the fat body protein content, which is relatively high in the second half of the last larval instar, but falls sharply during the final moult, and remains low in the first four to five days after the larval-adult ecdysis (Poels and Beenakkers, 1969). Thus, the subsequent increase of the fat body protein content, combined with the fairly constant specific enzyme activity, implies that the amount of total phosphorylase will increase during the adult stage.

The conversion of phosphorylase to its active form can also be achieved by the injection of extracts from larval CC (Table 4). Thus, the CC of fifth larval instar locusts are able to activate fat body glycogen phosphorylase, to elevate carbohydrate and lipid levels in haemolymph and, as has been demonstrated by Gäde and Beenakkers (1977), to raise cyclic AMP concentrations in the fat body. From these results it is obvious that the $\mathrm{CC}$ of larval locusts already possess the same potencies as are known for the $\mathrm{CC}$ of adult locusts.

That AKH actually has a physiological function in larval locusts and is not merely stored in the $\mathrm{CC}$ until in the adult stage the animals have developed flight capacity might be anticipated. The hormone could play a role during the larval-adult ecdysis, when fat body glycogen phosphorylase is strongly activated (Fig. 4). A similar phosphorylase activation has been observed in the fat body of Manduca sexta during the moult from the fourth to the fifth instar by Siegert and Ziegler (1983), who suggested that this activation depended on a hormonal factor from the $\mathrm{CC}$, the release of which would be induced by the reduced feeding activity during the moulting process.

Acknowledgements-The authors are indebted to $\mathrm{Mr} \mathrm{C} . \mathrm{M}$. Janmaat for maintaining the insect stock, to Mr J. J. van de Vlis for drawing the figures, and to Miss A. M. E. R. de Lange for typing the manuscript.

\section{REFERENCES}

Beenakkers A. M. Th. (1973) Influence of flight on lipid metabolism in Locusta migratoria. Insect Biochem. 3, 303-308.

Beenakkers A. M. Th., Van den Broek A. Th. M. and De Ronde Th. J. A. (1975) Development of catabolic pathways in insect flight muscles. A comparative study. $J$. Insect Physiol. 21, 849-859.

Beenakkers A. M. Th., Van der Horst D. J. and Van Marrewijk W. J. A. (1978) Regulation of release and metabolic function of the adipokinetic hormone in insects. In Comparative Endocrinology (Edited by Gaillard P. J. and Boer H. H.), pp. 445-448. Elsevier/ North-Holland Biomedical Press, Amsterdam.

Beenakkers A. M. Th., Van der Horst D. J. and Van Marrewijk W. J. A. (1981a) Metabolism during locust flight. Comp. Biochem. Physiol. 69B, 315-321.

Beenakkers A. M. Th., Van der Horst D. J. and Van Marrewijk W. J. A. (1981b) Role of lipids in energy metabolism. In Energy Metabolism and its Regulation in Insects (Edited by Downer R. G. H.), pp. 53-100. Plenum, New York.
Beenakkers A. M. Th., Van der Horst D. J. and Van Marrewijk W. J. A. (1984) Biochemical processes directed to flight muscle metabolism. In Comprehensive Insect Physiology, Biochemistry and Pharmacology (Edited by Kerkut G. A. and Gilbert L. I.), Vol. 10, Chap. 11. Pergamon Press, Oxford.

Gäde G. and Beenakkers A. M. Th. (1977) Adipokinetic hormone-induced lipid mobilization and cyclic AMP accumulation in the fat body of Locusta migratoria during development. Gen. comp. Endocr. 32, 481-487.

Goldsworthy G. J., Mordue W. and Guthkelch J. (1972) Studies on insect adipokinetic hormones. Gen. comp. Endocr. 18, 545-551.

Goldsworthy G. J., Mordue W. and Johnson R. A. (1973) The hormonal content of the locust corpus cardiacum. $J$. comp. Physiol. 85, 213-220.

Hill L. and Goldsworthy G. J. (1968) Growth, feeding activity, and the utilization of reserves in larvae of Locusta. J. Insect Physiol. 14, 1085-1098.

Jones J., Stone J. V. and Mordue W. (1977) The hyperglycaemic activity of locust adipokinetic hormone. Physiol. Ent. 2, 185-187.

Mayer R. J. and Candy D. J. (1969) Control of haemolymph lipid concentration during locust flight: an adipokinetic hormone from the corpora cardiaca. J. Insect Physiol. 15, 611-620.

Mwangi R. W. and Goldsworthy G. J. (1977) Age-related changes in the response to adipokinetic hormone in Locusta migratoria. Physiol. Ent. 2, 37-42.

Poels C. L. M. and Beenakkers A. M. Th. (1969) The effects of corpus allatum implantation on the development of flight muscles and fat body in Locusta migratoria. Entomologia exp. appl. 12, 312-324.

Schackterle G. R. and Pollack R. L. (1973) A simplified method for the quantitative assay of small amounts of protein in biological material. Analyt. Biochem. 51, 654-655.

Siegert K. and Ziegler R. (1983) A hormone from the corpora cardiaca controls fat body glycogen phosphorylase during starvation in tobacco hornworm larvae. Nature 301, 526-527.

Snedecor G. W. and Cochran W. G. (1967) Statistical Methods. Iowa State Univ. Press, Ames, lowa.

Sokal R. R. and Rohlf F. J. (1969) Biometry. Freeman, San Francisco.

Spik G. and Montreuil J. (1964) Deux causes d'erreur dans les dosages colorimétriques des oses neutres totaux. Bull. Soc. Chim. Biol. 46, 739-749.

Van der Horst D. J., Van Doorn J. M. and Beenakkers A. M. Th. (1978) Dynamics in the haemolymph trehalose pool during flight of the locust, Locusta migratoria. Insect Biochem. 8, 413-416.

Van der Horst D. J., Abbink J. H. M., Van Doorn J. M., Van Marrewijk W. J. A. and Beenakkers A. M. Th. (1983) Glycerol dynamics and metabolism during flight of the locust, Locusta migratoria. Insect Biochem. 13, 45-55.

Van Marrewijk W. J. A. and Beenakkers A. M. Th. (1982) Hormonal control of glycogenolysis in insect fat body directed to flight. Gen. comp. Endocr. 46, 380-381.

Van Marrewijk W. J. A., Van den Broek A. Th. M. and Beenakkers A. M. Th. (1980a) Regulation of glycogenolysis in the locust fat body during flight. Insect Biochem. 10, 675-679.

Van Marrewijk W. J. A., Schrikker A. E. M. and Beenakkers A. M. Th. (1980b) Contents of nucleic and amino acids and rate of protein synthesis in developing flight muscles of Locusta migratoria. Comp. Biochem. Physiol. 65B, 251-257.

Van Marrewijk W. J. A., Van den Broek A. Th. M. and Beenakkers A. M. Th. (1983) Regulation of glycogen phosphorylase activity in fat body of Locusta migratoria and Periplaneta americana. Gen. comp. Endocr. 50, 226-234. 
Van Norstrand M. D., Carlsen J. B., Josefsson L. and Herman W. S. (1980) Studies on a peptide with red pigment-concentrating and hyperglycemic activity from the cephalic endocrine system of the honeybee. Apis mellifera. Gen. comp. Endocr. 42, 526-533.

Wheeler C. H. and Goldsworthy G. J. (1983) Qualitative and quantitative changes in Locusta haemolymph pro- teins and lipoproteins during ageing and adipokinetic hormone action. J. Insect Physiol. 29, 339-347.

Zöllner N. and Kirsch K. (1962) Über die quantitative Bestimmung von Lipoîden (Mikromethode) mittels der vielen natürlichen Lipoîden (allen bekannten Plasmalipoïden) gemeinsamen SulfophosphovanillinReaktion. Z. ges. exp. Med. 135, 545-561. 